\title{
Surgery-related predictable risk factors influencing postoperative clinical outcomes for thoracic myelopathy caused by ossification of the posterior longitudinal ligament: a multicenter retrospective study
}

\author{
Hirokazu Saiwai, MD, PhD, ${ }^{1}$ Seiji Okada, MD, PhD, ${ }^{1,2}$ Mitsumasa Hayashida, MD, PhD, ${ }^{1}$ \\ Katsumi Harimaya, MD, PhD, ${ }^{3}$ Yoshihiro Matsumoto, MD, PhD, ${ }^{1}$ Ken-ichi Kawaguchi, MD, PhD, ${ }^{1}$ \\ Kazu Kobayakawa, MD, PhD, ${ }^{4}$ Takeshi Maeda, MD, PhD, ${ }^{4}$ Hideki Ohta, MD, ${ }^{5}$ Kenzo Shirasawa, MD, ${ }^{6}$ \\ Kuniyoshi Tsuchiya, MD, PhD, ${ }^{7}$ Kazumasa Terada, MD, PhD, ${ }^{8}$ Kouzo Kaji, MD, ${ }^{9}$ \\ Takeshi Arizono, MD, PhD, ${ }^{10}$ Taichi Saito, MD, PhD, ${ }^{11}$ Masami Fujiwara, MD, ${ }^{12}$ \\ Yukihide Iwamoto, MD, PhD, ${ }^{9}$ and Yasuharu Nakashima, MD, PhD1
}

\begin{abstract}
'Department of Orthopedic Surgery, Graduate School of Medical Sciences; '2Department of Immunobiology and Neuroscience, Medical Institute of Bioregulation, Kyushu University, Fukuoka; ${ }^{3}$ Department of Orthopedic Surgery, Kyushu University Beppu Hospital, Oita; ${ }^{4}$ Department of Orthopedic Surgery, Spinal Injuries Center, Fukuoka; ${ }^{5}$ Oita Orthopedic Hospital, Oita; ${ }^{6}$ Shimonoseki City Hospital, Yamaguchi; ' 7 Department of Orthopedic Surgery, Japan Community Health Care Organization, Kyushu Hospital, Fukuoka; ${ }^{8}$ Department of Orthopedic Surgery, National Hospital Organization, Kyushu Medical Center, Fukuoka; ' ${ }^{D}$ epartment of Orthopedic Surgery, Kyushu Rosai Hospital, Fukuoka; ${ }^{10}$ Department of Orthopedic Surgery, Kyushu Central Hospital, Fukuoka; ${ }^{11}$ Department of Orthopedic Surgery, Fukuoka City Hospital, Fukuoka; and ${ }^{12}$ Department of Orthopedic Surgery, Sada Hospital, Fukuoka, Japan
\end{abstract}

OBJECTIVE Compression of the spinal cord by thoracic ossification of the posterior longitudinal ligament (T-OPLL) often causes severe thoracic myelopathy. Although surgery is the most effective treatment for T-OPLL, problems associated with surgical intervention require resolution because surgical outcomes are not always favorable, and a small number of patients experience deterioration of their neurological status after surgery. The aim of the present study was to examine the surgery-related risk factors contributing to poor clinical outcomes for myelopathy caused by T-OPLL.

METHODS Data were extracted from the records of 55 patients with thoracic myelopathy due to T-OPLL at institutions in the Fukuoka Spine Group. The mean follow-up period was 5.3 years. Surgical outcomes were assessed using the Japanese Orthopaedic Association (JOA) scale. To investigate the definitive factors associated with surgical outcomes, univariate and multivariate regression analyses were performed with several patient-related and surgery-related factors, including preoperative comorbidities, radiological findings, JOA score, surgical methods, surgical outcomes, and complications.

RESULTS Neurological status improved in 33 patients (60.0\%) and deteriorated in 10 patients (18.2\%) after surgery. The use of instrumentation was significantly associated with an improved outcome. In the comparison of surgical approaches, posterior decompression and fusion resulted in a significantly higher neurological recovery rate than did anterior decompression via a posterior approach and fusion or decompression alone. It was also found that postoperative neurological status was significantly poorer when there were fewer instrumented spinal levels than decompression levels. CSF leakage was a predictable risk factor for deterioration following surgery.

CONCLUSIONS It is important to identify preventable risk factors for poor surgical outcomes for T-OPLL. The findings of the present study suggest that intraoperative CSF leakage and a lower number of instrumented spinal fusion levels than decompression levels were exacerbating factors for the neurological improvement in T-OPLL surgery.

https://thejns.org/doi/abs/10.3171/2019.10.SPINE19831

KEYWORDS thoracic ossification of the posterior longitudinal ligament; thoracic myelopathy; cerebrospinal fluid leak; posterior decompression and fusion surgery; surgery-related predictable risk factors

ABBREVIATIONS JOA = Japanese Orthopaedic Association; OLF = ossification of the ligamentum flavum; OPLL = ossification of the posterior longitudinal ligament; T-OPLL = thoracic OPLL.

SUBMITTED July 17, 2019. ACCEPTED October 28, 2019.

INCLUDE WHEN CITING Published online December 27, 2019; DOI: 10.3171/2019.10.SPINE19831. 
$\mathrm{T}$ HORACIC Ossification of the posterior longitudinal ligament (T-OPLL) is a relatively rare condition but a well-known cause of severe thoracic myelopathy. Following the development of symptomatic myelopathy due to spinal cord compression by T-OPLL, conservative treatment, including rest or immobilization with a brace, has been considered to be ineffective, and surgical treatment is required to obtain symptomatic improvement. .,17,18 $^{-1}$ The aim of the T-OPLL surgery is to decompress the spinal cord to prevent the progression of myelopathy. However, the surgical outcomes of T-OPLL are generally unfavorable compared with those of cervical OPLL. ${ }^{20,29}$ This is due to the presence of several anatomical and clinical features that render T-OPLL surgery difficult. First, although direct decompression of the T-OPLL is theoretically the optimal procedure, it is technically difficult to remove the ossification via an anterior or posterior approach due to the anatomical location of the PLL. Second, the ossified ligament is often adhered to the dural sac, which may result in CSF leakage. Third, posterior decompression is less effective due to the kyphotic alignment of the thoracic spine. Fourth, the spinal cord at the thoracic level is particularly fragile and prone to damage by the surgical procedure. Therefore, although surgery is the most effective treatment for T-OPLL, problems associated with surgical intervention require resolution because surgical outcomes are not always favorable, and the neurological status of a small number of patients declines following surgery. ${ }^{1,6,17,26}$ The purpose of this multicenter retrospective study was to evaluate the surgical outcomes and factors associated with a poor prognosis following T-OPLL surgery.

\section{Methods}

Data were extracted from the medical records and radiological images of 55 patients with thoracic myelopathy caused by T-OPLL, who underwent surgery between 2000 and 2010 at institutions where members of the Fukuoka Spine Group practice. Of these patients, 30 were females and 25 were males, and their mean age was 56.0 years (range 31-76 years) at the time of surgery. The mean follow-up period was 5.3 years (range $0.6-15.4$ years).

The following patient data were obtained: disease duration, BMI, comorbidities, ossification type, anteroposterior diameter of the ossification, maximum level of the ossification, surgical methods, decompression and fusion levels, surgical time, total blood loss, and complications. Statistical analyses were performed to determine the critical prognostic factors associated with surgical outcomes.

Patients with a BMI between 25 and $29.9 \mathrm{~kg} / \mathrm{m}^{2}$ were classified as overweight, and those with a BMI $>30 \mathrm{~kg} / \mathrm{m}^{2}$ were classified as obese. Morphological measurements and the level of the ossified ligament, including ossification of the ligamentum flavum (OLF) and OPLL, were determined on lateral radiography and/or CT and/or MRI. T-OPLL was morphologically classified into 5 types: linear, beaked, continuous waveform, continuous cylindrical, and mixed. ${ }^{19}$

The modified Japanese Orthopaedic Association (JOA) scoring system for thoracic myelopathy (maximum 11 points) and the modified Frankel grading system ${ }^{5,9}$ were used to evaluate the clinical outcomes of patients, preand postoperatively. The recovery rates were calculated according to the following equation: (postoperative JOA score - preoperative JOA score)/(11 points - preoperative JOA score) $\times 100(\%){ }^{11}$

\section{Statistical Analysis}

JMP Pro 11.0.0 software (SAS Institute) was used to perform all statistical analyses. The Wilcoxon rank-sum test was used to compare the medians of the JOA score prior to and following surgery as well as the recovery rate of the JOA score, age at surgery, body height, body weight, BMI, disease duration, anteroposterior diameter of the ossification, surgical time, total blood loss, and decompression range. Univariate and multivariate linear regression analyses were performed to identify the independent predictors of the recovery rate of the JOA score. The chi-square test was used to compare groups regarding categorical variables. All tests were two-sided, and the level of significance was set at 0.05 . The data are presented as the mean \pm standard deviation.

\section{Results}

\section{Clinical Data}

In the study cohort, the mean BMI was $28.6 \mathrm{~kg} / \mathrm{m}^{2}$ (range $19.0-45.8 \mathrm{~kg} / \mathrm{m}^{2}$ ). According to the BMI categories, 22 patients $(40.0 \%)$ were considered overweight and 16 patients were obese $(29.1 \%)$. A total of 36 patients $(65.5 \%)$ had comorbid conditions. Among these, 14 patients $(25.5 \%)$ had more than 2 comorbidities; 16 patients (29.1\%) had diabetes mellitus, 16 patients $(29.1 \%)$ had hypertension, and 6 patients $(10.9 \%)$ had liver disorder (Table 1).

\section{Radiological Data}

The T-OPLL type was classified as beaked in 24 patients (43.6\%), continuous waveform in 14 patients $(25.5 \%)$, continuous linear in 2 patients $(3.6 \%)$, and mixed in 17 patients $(30.9 \%)$. The average number of vertebral levels of OPLL was 6.3 (range 2-22). The maximum ossification was detected between $\mathrm{T} 1$ and $\mathrm{T} 4$ in 23 patients $(41.8 \%)$, T5 and T8 in 21 patients $(38.2 \%)$, and T9 and T12 in 11 patients $(20.0 \%)$ (Table 1$)$. Thirty-one patients $(56.4 \%)$ had OLF at the thoracic spine (27 patients), at the thoracic and lumbar spine (3 patients), and at the cervical and thoracic spine (1 patient). Among these, 22 patients $(40.0 \%)$ had simultaneous ossification of the OPLL and OLF at the same vertebral level of the thoracic spine. The mean anteroposterior diameter of the spinal canal at the level of maximum ossification was $7.8 \mathrm{~mm}$ (range $4.0-18.0 \mathrm{~mm}$ ).

\section{Surgical Outcomes}

The surgical plan was determined based on preoperative neurological status and findings from imaging, including radiography, MRI, myelography, and CT myelography. Posterior decompression was performed in 12 patients, posterior decompression and fusion with instrumentation was performed in 33 patients, and anterior decompression via a posterior approach, reported by Ohtsuka et al., ${ }^{21}$ was 
TABLE 1. Demographic and clinical data obtained in 55 patients with T-OPLL

\begin{tabular}{cc}
\hline & Value \\
\hline Age at surgery, yrs & $56.0 \pm 12.3$ \\
\hline Sex, male/female & $25: 30$ \\
\hline Body height, cm & $160.2 \pm 10.0$ \\
\hline Body weight, kg & $74.1 \pm 21.5$ \\
\hline BMl, kg/m ${ }^{2}$ & $28.6 \pm 6.4$ \\
\hline Comorbidity present & $65.5 \%(36)$ \\
\hline Diabetes mellitus & $29.1 \%(16)$ \\
\hline Hypertention & $29.1 \%(16)$ \\
\hline Liver disorder & $10.9 \%(6)$ \\
\hline Ossification type & \\
\hline Beaked & $43.6 \%(24)$ \\
\hline Continuous waveform & $25.5 \%(14)$ \\
\hline Continuous linear & $3.6 \%(2)$ \\
\hline Mixed & $30.9 \%(17)$ \\
\hline Maximum ossification level & \\
\hline T1-4 & $41.8 \%(23)$ \\
\hline T5-8 & $38.2 \%(21)$ \\
\hline T9-12 & $20.0 \%(11)$ \\
\hline OLF present & $56.4 \%(31)$ \\
\hline
\end{tabular}

Values are presented as the mean \pm SD or percentage (number) of patients.

performed in 10 patients. The mean JOA score prior to surgery was 4.14 (range $0-9$ ), and the score following surgery was 5.93 (range $0-11$ ). The recovery rate was $\geq 50 \%$ in 20 patients $(36.4 \%)$ and $\geq 25 \%$ in 30 patients $(54.5 \%)$. Neurological status improved in 33 patients $(60.0 \%)$, remained unchanged in 12 patients $(21.8 \%)$, and deteriorated in 10 patients (18.2\%) according to the Modified Frankel grading system. ${ }^{9}$

One or more major surgery-related complications occurred in 31 patients $(56.4 \%)$, including neurological deterioration, CSF leakage, clinically significant epidural hematoma, and surgical site infection. CSF leakage occurred in 14 patients $(25.5 \%)$. Of the 14 patients with CSF leakage, 7 had neurological deterioration. Postoperative epidural hematomas occurred in 5 patients, and 3 of the 5 patients required surgical intervention and evacuation of hematoma immediately following the initial surgery. Postoperative surgical site infection occurred in 1 patient, who required additional surgery to irrigate the infection site (Table 2).

\section{Clinical Data and Complications Related to Surgical Outcomes}

Based on the univariate linear regression analysis, the recovery rate of the JOA score was significantly associated with instrumentation use ( $\beta 21.15,95 \%$ CI 8.09-34.22), postoperative hematoma ( $\beta-27.39,95 \% \mathrm{CI}-47.41$ to -7.37), and CSF leakage at the time of surgery $(\beta-16.59$, $95 \%$ CI -29.75 to -3.44 ). None of the patients' demographic factors were significantly associated with the recovery rate. Multivariate linear regression analysis was also
TABLE 2. Surgical outcomes

\begin{tabular}{lc}
\hline & Value \\
\hline \multicolumn{2}{l}{ Surgical method } \\
\hline Posterior decompression & $21.8 \%(12)$ \\
\hline $\begin{array}{l}\text { Posterior decompression \& fusion w/ instrumenta- } \\
\quad \text { tion }\end{array}$ & $60.0 \%(33)$ \\
\hline Anterior decompression via a posterior approach & $18.2 \%(10)$ \\
\hline Mean preop JOA score & 4.14 \\
\hline Mean postop JOA score & 5.93 \\
\hline JOA recovery rate $>50 \%$ & $36.4 \%(20)$ \\
\hline JOA recovery rate $>25 \%$ & $54.5 \%(30)$ \\
\hline Modified Frankel grade & \\
\hline Improved & $60.0 \%(33)$ \\
\hline Unchanged & $21.8 \%(12)$ \\
\hline Deteriorated & $18.2 \%(10)$ \\
\hline Surgery-related complications & $56.4 \%(31)$ \\
\hline CSF leak & $25.5 \%(14)$ \\
\hline Epidural hematoma & $9.1 \%(5)$ \\
\hline Surgical site infection & $1.8 \%(1 / 55)$ \\
\hline
\end{tabular}

Values are presented as the percentage (number) of patients unless stated otherwise.

performed to remove confounding factors, the results of which showed that the use of instrumentation $(\beta 23.03$, 95\% CI 2.42-43.64) and CSF leakage ( $\beta-24.93,95 \%$ CI -47.27 to -2.59 ) were independent predictors of the rate of JOA score recovery (Table 3). To further investigate the effect of use of instruments for postoperative neurological improvement, the patients were divided into 3 groups according to the surgical methods: posterior decompression and fusion group $(\mathrm{n}=33)$, anterior decompression via posterior approach and fusion group $(\mathrm{n}=10)$, and decompression-only group $(n=12)$. The modified Frankel grade was significantly improved postoperatively in the posterior decompression and fusion group compared with the other groups (Table 4). Among 33 patients in the posterior decompression and fusion group, 5 patients had posterior decompression and partial spinal fusion. These patients had a significantly poorer outcome according to the JOA score than those who had at least as many levels of fusion as levels of decompression (Table 5).

CSF leakage was an independent predisposing factor for exacerbation of JOA score recovery rate in the present study (Table 3). To investigate the risk factors for CSF leak, several patient-related and surgery-related factors were compared in patients with or without CSF leak. Among these factors, surgical time was the only significant factor that was statistically associated with CSF leak (Table 6). No other factors such as the shape or morphology of the OPLL or the type of surgical procedure were associated with CSF leak in this study. However, CSF leakage significantly exacerbated neurological recovery following surgery (Table 7).

\section{Morphological Change of OPLL in Representative Cases}

Preoperative and postoperative CT images of a repre- 
TABLE 3. Multivariate linear regression analysis of the JOA score recovery rate

\begin{tabular}{lcc}
\hline \multicolumn{1}{c}{ Variable } & $\beta(95 \%$ Cl $)$ & $p$ Value \\
\hline Age at surgery & $0.30(-1.21$ to 1.82$)$ & 0.69 \\
\hline Male sex & $-2.24(-24.02$ to 19.53$)$ & 0.83 \\
\hline Body height & $3.65(-3.14$ to 10.43$)$ & 0.28 \\
\hline Body weight & $-3.07(-9.78$ to 3.64$)$ & 0.36 \\
\hline BMl & $9.34(-8.91$ to 27.59$)$ & 0.30 \\
\hline Diabetes mellitus & $-0.63(-5.14$ to 3.88$)$ & 0.78 \\
\hline Hypertension & $-15.93(-35.37$ to 3.52$)$ & 0.10 \\
\hline Disease duration & $5.51(-19.82$ to 30.83$)$ & 0.66 \\
\hline Beak-type OPLL & $1.34(-13.19$ to 15.88$)$ & 0.85 \\
\hline AP diameter of ossification & $-0.16(-8.21$ to 7.88$)$ & 0.97 \\
\hline Preop JOA score & $2.71(-5.18$ to 10.60$)$ & 0.49 \\
\hline Surgical time & $-0.07(-0.25$ to 0.11$)$ & 0.42 \\
\hline Total blood loss & $0.03(-0.01$ to 0.06$)$ & 0.17 \\
\hline Use of instrumentation & $23.03(2.42$ to 43.64$)$ & 0.030 \\
\hline Postop hematoma & $-6.65(-31.91$ to 18.61$)$ & 0.59 \\
\hline CSF leak at operation & $-24.93(-47.27$ to -2.59$)$ & 0.030 \\
\hline
\end{tabular}

$A P=$ anteroposterior $\beta=$ unstandardized regression coefficient. $\mathrm{R}^{2}=0.51$.

sentative case with a good outcome are shown in Fig. 1A and $\mathrm{B}$. The mixed type of OPLL at the middle thoracic level was observed on the preoperative image, and the OPLL contained the nonossified segment (Fig. 1A). The postoperative CT image showed the bony fusion of the nonossified segment of OPLL (Fig. 1B), which contributes to a better outcome by obtaining a more stable condition of the spinal cord. CT images obtained in a case with poor outcome are shown in Fig. 1C and D. The patient had cervical and thoracic mixed-type OPLL, and the nonossified segment was observed on the preoperative image at C7-T1 and T2-3 (Fig. 1C). We performed cervical laminoplasty and thoracic posterior decompression and fusion surgery in this case. Since the instrumented spinal fusion was limited to T1-7, the C7-T1 segment showed adjacent-segment disease and significant OPLL growth, which would be the main reason for the neurological deterioration. The preoperative nonossified segment at T2-3 showed bony fusion after surgery since these segments were included in the fusion level (Fig. 1D).

\section{Discussion}

The present study revealed 4 major findings relating to
T-OPLL surgery. First, posterior decompression and fusion surgery resulted in superior neurological recovery compared with anterior decompression via a posterior approach and fusion or decompression alone. Second, the postoperative neurological status was significantly poorer when the number of instrumented spinal levels was lower than that of decompression levels. Third, intraoperative CSF leakage was significantly associated with the deterioration of postoperative neurological function. Finally, any of the patient-related and surgery-related factors, with the exception of surgical time, were not associated with intraoperative CSF leakage. These results contribute to reducing the risk of surgical deterioration of thoracic myelopathy due to T-OPLL.

The main purpose of T-OPLL surgery is to decompress the spinal cord from the narrow spinal canal to improve myelopathy or prevent its progression. However, the importance of spinal fusion in addition to the spinal cord decompression has been emphasized for T-OPLL surgery in previous reports due to the biomechanical effects of spinal fusion, including correcting kyphosis, stabilizing the spine, and maintaining spinal alignment of the decompression level. ${ }^{27-29}$ The proposed mechanism of postoperative neurological deterioration following surgery with decompression alone is the progression of thoracic kyphosis and OPLL growth at the level of surgery, resulting in the spinal cord being compressed from anteriorly by OPLL. ${ }^{13,25}$

In the present study, 2 types of fusion surgery were performed. The first one comprises posterior decompression and fusion and the second comprises anterior decompression via a posterior approach and fusion. The clinical outcomes were significantly improved in the posterior decompression and fusion group compared with those in the anterior decompression via a posterior approach and fusion surgery group. Anterior decompression via a posterior approach and fusion was investigated by Ohtsuka et al., ${ }^{21}$ allowing direct decompression of the spinal cord. However, this method is technically demanding compared with posterior decompression and fusion, which would risk poorer surgical outcomes in this study. Takahata et al. examined postoperative outcomes in 30 cases of anterior decompression via a posterior approach and fusion for T-OPLL and reported that 10 of 30 patients had postoperative neurological deterioration, ${ }^{24}$ which was a higher incident rate than that in other reports using different surgical methods for the treatment of T-OPLL.16,28 Therefore, although spinal cord decompression is indirect, posterior decompression and fusion has an advantage due to its simpler and safer procedure, leading to more favorable outcomes. Furthermore, there are several reports of the ben-

TABLE 4. Association between spinal fixation and symptomatic improvement

\begin{tabular}{lcccc}
\hline & \multicolumn{3}{c}{ Change in Modified Frankel Grade } & \\
\cline { 2 - 4 } & Improved & Unchanged & Exacerbated & $p$ Value* \\
\hline Posterior decompression \& fusion $(n=33)$ & 25 & 7 & 1 & \\
\hline Anterior decompression via posterior approach \& fusion $(n=10)$ & 4 & 2 & 4 & \\
\hline Decompression only $(n=12)$ & 4 & 3 & 5 & \\
\hline${ }^{*}$ Chi-square test. & & &
\end{tabular}


TABLE 5. Association between fixation range and outcome of the surgery

\begin{tabular}{lccc}
\hline & $\begin{array}{c}\text { No. of Fixation Levels } \geq \text { Decompression } \\
\text { Levels }\end{array}$ & $\begin{array}{c}\text { No. of Fixation Levels < Decompression } \\
\text { Levels }\end{array}$ & p Value* \\
\hline JOA score before surgery & $4.03 \pm 1.90$ & $3.8 \pm 1.92$ & 0.42 \\
\hline JOA score after surgery & $7.41 \pm 2.20$ & $4.2 \pm 3.11$ & 0.04 \\
\hline JOA recovery rate, $\%$ & $48.53 \pm 27.55$ & $7.85 \pm 27.47$ & 0.017 \\
\hline
\end{tabular}

Values are presented as the mean \pm SD unless stated otherwise.

${ }^{*}$ Mann-Whitney U-test.

eficial effect of instrumentation for the ossified ligament biology; spinal instrumentation resulted in bony fusion of the nonossified interstice between the segmental OPLLs and led to a reduction in the thickness of the ossified ligament. ${ }^{3,14,15}$

In general, spinal fusion has several potential risks, including infection, implant failure, and adjacent-segment disease, and these risks increase as the number of instrumented spinal levels increases. To minimize these risks, 5 cases in the present study had a lower number of fusion levels than decompression levels in this series, which resulted in significantly poorer clinical outcomes compared with the 27 cases with longer fusion levels than decompression levels or the same fusion levels as decompression levels. The possible mechanism for this result is a lack of the biomechanical effects of spinal fusion described above at the levels of decompression without fusion.

As the results of the present study suggested, CSF leakage is a significant factor for postoperative neurological deterioration; it is important to avoid intraoperative dural tear. However, no association between the occurrence of CSF leakage and patient-related factors or surgery-related factors was identified, with the exception of surgical time in the present study, suggesting that CSF leakage may occur sporadically in any situation during initial surgery and subsequent surgical interventions. Therefore, close attention is required to prevent CSF leakage during decompression procedures as part of T-OPLL surgery.

As previously reported, ${ }^{23}$ the Kerrison punch is the instrument that is most often used when dural tearing occurs. When we perform laminectomy for cases of TOPLL, we can hardly retract the dura mater since such retraction would cause spinal cord injury at the thoracic level. Therefore, it is difficult to observe the footplate of the Kerrison punch when it is inserted under the lamina or ligamentum flavum. We think that this is the main reason for the high risk of dural tear caused when using the Kerrison punch. To prevent dural tearing by the Kerrison punch, we recently used a high-speed diamond burr to thin the lamina as much as possible before using the Kerrison punch. Extremely thinned lamina is pliable enough to elevate it using the footplate of the Kerrison punch and create some space between lamina and underlying dura mater even though the dura mater is not retracted. Then, we can insert the Kerrison punch safely under the thinned lamina before biting it. In addition, we can easily check whether or not the lamina adheres to the dura when we insert the punch into the space because, if the punch touches dura mater that is adhered to the lamina or surrounding tissues, the exposed dura moves in accordance with the motion of the Kerrison punch; thus, it is a sign of dural adhesion and has a higher risk of dural tear.

As we showed that CSF leakage is associated with worse outcomes, reduction of the incidence of dural tears is the most effective and feasible approach to obtain better outcomes not only for T-OPLL surgery but also for other spine surgeries in which decompression is performed.

Several pathophysiological mechanisms have been suggested for the cause of neurological deterioration as a result of CSF leakage during T-OPLL surgery. These mechanisms are associated with anatomical features of the thoracic spine and spinal cord, particularly CSF function and blood flow. T-OPLL develops the most frequently at the middle thoracic spine where the spinal canal is

TABLE 6. Relationships between CSF leakage and other variables

\begin{tabular}{lccc}
\hline \multicolumn{1}{c}{ Variable } & Patients w/ CSF Leak $(n=14)$ & Patients w/o CSF Leak $(n=41)$ & $p$ Value \\
\hline Age at surgery, yrs & $58.6 \pm 3.2$ & $55.1 \pm 1.9$ & 0.28 \\
\hline Body height, $\mathrm{cm}$ & $161.1 \pm 2.1$ & $159.9 \pm 1.6$ & 0.58 \\
\hline Body weight, $\mathrm{kg}$ & $74.6 \pm 4.8$ & $74.0 \pm 3.5$ & 0.55 \\
\hline BMl & $28.7 \pm 1.6$ & $28.6 \pm 1.0$ & 0.60 \\
\hline Disease duration, $\mathrm{yr}$ & $3.7 \pm 1.6$ & $1.5 \pm 0.3$ & 0.49 \\
\hline AP diameter of ossification, $\mathrm{mm}$ & $8.3 \pm 1.0$ & $7.5 \pm 0.3$ & 0.99 \\
\hline JOA score before surgery & $4.14 \pm 0.54$ & $4.15 \pm 0.32$ & 0.96 \\
\hline Surgical time, mins & $326.1 \pm 34.1$ & $245.4 \pm 14.3$ & 0.021 \\
\hline Total blood loss, $\mathrm{ml}$ & $726.0 \pm 202.5$ & $511.7 \pm 65.5$ & 0.40 \\
\hline
\end{tabular}

Values are presented as the mean \pm SD unless stated otherwise.

* Wilcoxon rank-sum test. 
TABLE 7. Effect of CSF leakage on neurological function

\begin{tabular}{lcccc}
\hline & \multicolumn{3}{c}{ Change in Modified Frankel Grade } & \\
\cline { 2 - 4 } & Improved & Unchanged & Worsened & p Value* \\
\hline $\begin{array}{c}\text { Patients w/ CSF leak } \\
(n=14)\end{array}$ & 4 & 6 & 4 & \\
$\begin{array}{l}\text { Patients w/o CSF leak } \\
(n=41)\end{array}$ & 30 & 6 & 5 & \\
\hline
\end{tabular}

${ }^{*}$ Chi-square test.

the narrowest. The spinal cord blood flow is also poor at this level. ${ }^{4}$ To protect the spinal cord from the mechanical pressure by surrounding environments, CSF serves an important role by providing hydromechanical support. ${ }^{22}$ Once a dural tear occurs, CSF leakage begins, which leads to the loss of hydromechanical protection and may exacerbate the susceptibility of spinal cord compression from the surrounding soft tissue and postoperative hematomas. A CSF leak also results in an increased compression at the ventral side of thoracic spinal cord due to OPLL, leading to ischemic injury of the spinal cord due to aggravation of the tenuous arterial blood supply and venous drainage of the spinal cord..$^{8,10}$

In the present study, posterior decompression and fusion surgery led to improved outcomes. However, selection of the most appropriate surgical methods depending on the patients' conditions is required. Decompression with corrective fusion of the thoracic spine has been reported to be an effective method for spinal cord decompression since the spinal cord can be shifted posteriorly by the procedure. ${ }^{2,30}$ This technique may assist in expanding the indication for posterior fusion and decompression surgery, which is relatively safe and less technically demanding. However, the indication of dekyphosis and the degree of reduction by the dekyphosis are still controversial since decompression with corrective fusion may cause instrument failure or loosening of pedicle screws. ${ }^{12,16}$

The present study has several limitations. First, it was a retrospective study. Second, the number of patients was relatively small. Third, the surgical outcomes were ob- tained from only 3 surgical methods. However, it was identified that intraoperative CSF leakage and a lower number of instrumented spinal fusion than decompression levels were significant risk factors for postoperative neurological deterioration in T-OPLL surgery.

\section{Conclusions}

It is important to identify preventable risk factors for poor surgical outcomes for T-OPLL. The findings of the present study suggest that intraoperative CSF leakage and a lower number of instrumented spinal fusion levels than decompression levels were exacerbating factors for the neurological improvement in T-OPLL surgery.

\section{References}

1. Aizawa T, Sato T, Sasaki H, Matsumoto F, Morozumi N, Kusakabe T, et al: Results of surgical treatment for thoracic myelopathy: minimum 2-year follow-up study in 132 patients. J Neurosurg Spine 7:13-20, 2007

2. Ando K, Imagama S, Ito Z, Kobayashi K, Ukai J, Muramoto A, et al: Ponte osteotomy during dekyphosis for indirect posterior decompression with ossification of the posterior longitudinal ligament of the thoracic spine. Clin Spine Surg 30:E358-E362, 2017

3. Ando K, Imagama S, Ito Z, Kobayashi K, Ukai J, Muramoto A, et al: Radiologic evaluation after posterior instrumented surgery for thoracic ossification of the posterior longitudinal ligament: union between rostral and caudal ossifications. J Spinal Disord Tech 27:181-184, 2014

4. Dommisse GF: The blood supply of the spinal cord. A critical vascular zone in spinal surgery. J Bone Joint Surg Br 56:225-235, 1974

5. Frankel HL, Hancock DO, Hyslop G, Melzak J, Michaelis LS, Ungar GH, et al: The value of postural reduction in the initial management of closed injuries of the spine with paraplegia and tetraplegia. I. Paraplegia 7:179-192, 1969

6. Fujimura Y, Nishi Y, Nakamura M, Toyama Y, Suzuki N: Long-term follow-up study of anterior decompression and fusion for thoracic myelopathy resulting from ossification of the posterior longitudinal ligament. Spine (Phila Pa 1976) 22:305-311, 1997

7. Fujimura Y, Nishi Y, Nakamura M, Watanabe M, Matsumoto M: Myelopathy secondary to ossification of the posterior
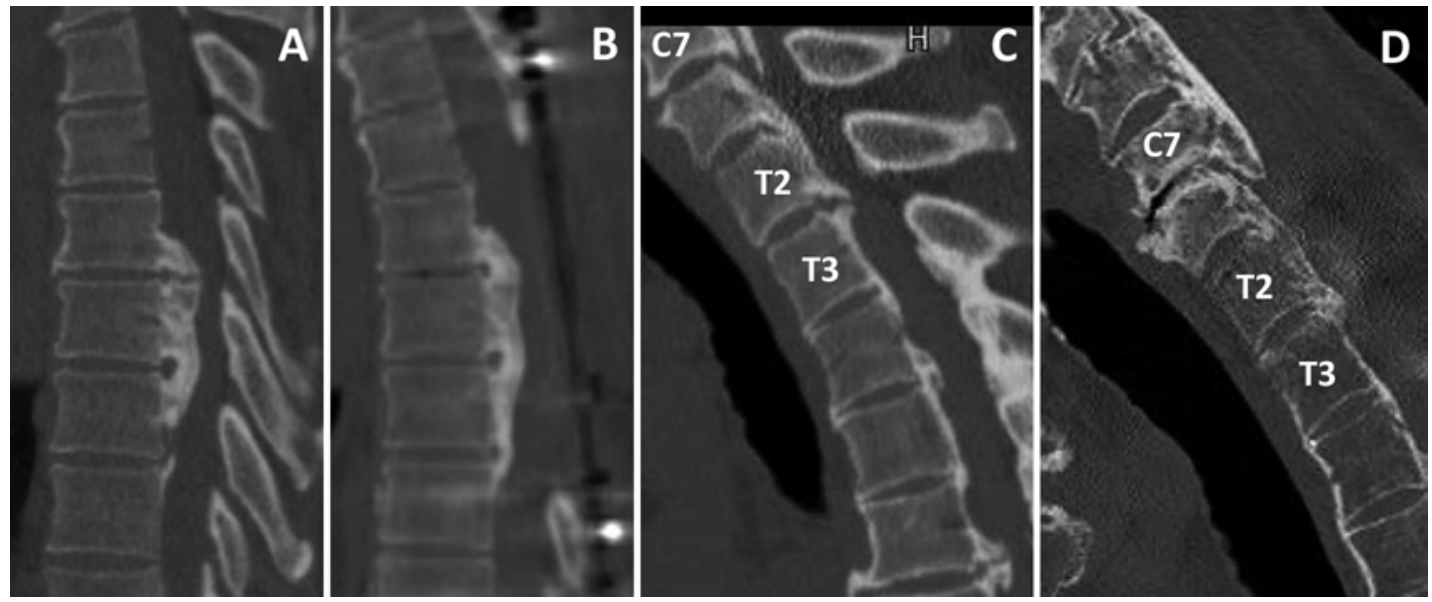

FIG. 1. Pre- and postoperative CT images of cases with a good outcome (A and B) and a poor outcome (C and D). A and C: Preoperative sagittal images. B and D: Postoperative sagittal images. 
longitudinal ligament of the thoracic spine treated by anterior decompression and bony fusion. Spinal Cord 35:777-784, 1997

8. Griessenauer CJ, Raborn J, Foreman P, Shoja MM, Loukas M, Tubbs RS: Venous drainage of the spine and spinal cord: a comprehensive review of its history, embryology, anatomy, physiology, and pathology. Clin Anat 28:75-87, 2015

9. Hayashi T, Kawano O, Sakai H, Ideta R, Ueta T, Maeda T, et al: The potential for functional recovery of upper extremity function following cervical spinal cord injury without major bone injury. Spinal Cord 51:819-822, 2013

10. Henderson FC, Crockard HA, Stevens JM: Spinal cord oedema due to venous stasis. Neuroradiology 35:312-315, 1993

11. Hirabayashi K, Miyakawa J, Satomi K, Maruyama T, Wakano K: Operative results and postoperative progression of ossification among patients with ossification of cervical posterior longitudinal ligament. Spine (Phila Pa 1976) 6:354-364, 1981

12. Hyun SJ, Lee BH, Park JH, Kim KJ, Jahng TA, Kim HJ: Proximal junctional kyphosis and proximal junctional failure following adult spinal deformity surgery. Korean J Spine 14:126-132, 2017

13. Katsumi K, Watanabe K, Izumi T, Hirano T, Ohashi M, Mizouchi T, et al: Natural history of the ossification of cervical posterior longitudinal ligament: a three dimensional analysis. Int Orthop 42:835-842, 2018

14. Kimura H, Fujibayashi S, Takemoto M, Otsuki B, Matsuda $\mathrm{S}$ : Spontaneous reduction in ossification of the posterior longitudinal ligament of the thoracic spine after posterior spinal fusion without decompression: a case report. Spine (Phila Pa 1976) 39:E417-E419, 2014

15. Koda M, Furuya T, Okawa A, Aramomi M, Inada T, Kamiya $\mathrm{K}$, et al: Bone union and remodelling of the non-ossified segment in thoracic ossification of the posterior longitudinal ligament after posterior decompression and fusion surgery. Eur Spine J 24:2555-2559, 2015

16. Koda M, Furuya T, Okawa A, Inada T, Kamiya K, Ota M, et al: Mid- to long-term outcomes of posterior decompression with instrumented fusion for thoracic ossification of the posterior longitudinal ligament. J Clin Neurosci 27:87-90, 2016

17. Matsumoto M, Chiba K, Toyama Y, Takeshita K, Seichi A, Nakamura K, et al: Surgical results and related factors for ossification of posterior longitudinal ligament of the thoracic spine: a multi-institutional retrospective study. Spine (Phila Pa 1976) 33:1034-1041, 2008

18. Matsuyama Y, Yoshihara H, Tsuji T, Sakai Y, Yukawa Y, Nakamura H, et al: Surgical outcome of ossification of the posterior longitudinal ligament (OPLL) of the thoracic spine: implication of the type of ossification and surgical options. J Spinal Disord Tech 18:492-498, 2005

19. Mori K, Imai S, Kasahara T, Nishizawa K, Mimura T, Matsusue Y: Prevalence, distribution, and morphology of thoracic ossification of the posterior longitudinal ligament in Japanese: results of CT-based cross-sectional study. Spine (Phila Pa 1976) 39:394-399, 2014

20. Ohtani K, Nakai S, Fujimura Y, Manzoku S, Shibasaki K: Anterior surgical decompression for thoracic myelopathy as a result of ossification of the posterior longitudinal ligament. Clin Orthop Relat Res (166):82-88, 1982

21. Ohtsuka K, Terayama K, Tsuchiya T, Wada K, Furukawa K A surgical procedure for the anterior decompression of the spinal cord through the posterior approach. Orthop Surg Traumatol 36:1083-1090, 1983 (Japanese)
22. Sakka L, Coll G, Chazal J: Anatomy and physiology of cerebrospinal fluid. Eur Ann Otorhinolaryngol Head Neck Dis 128:309-316, 2011

23. Sin AH, Caldito G, Smith D, Rashidi M, Willis B, Nanda A: Predictive factors for dural tear and cerebrospinal fluid leakage in patients undergoing lumbar surgery. J Neurosurg Spine 5:224-227, 2006

24. Takahata M, Ito M, Abumi K, Kotani Y, Sudo H, Minami A: Clinical results and complications of circumferential spinal cord decompression through a single posterior approach for thoracic myelopathy caused by ossification of posterior longitudinal ligament. Spine (Phila Pa 1976) 33:1199-1208, 2008

25. Tokuhashi Y, Matsuzaki H, Oda H, Uei H: Effectiveness of posterior decompression for patients with ossification of the posterior longitudinal ligament in the thoracic spine: usefulness of the ossification-kyphosis angle on MRI. Spine (Phila Pa 1976) 31:E26-E30, 2006

26. Tomita K: Total decompression of the spinal cord for combined ossification of posterior longitudinal ligament and yellow ligament in the thoracic spine. Arch Orthop Trauma Surg 109:57-62, 1990

27. Yamazaki M, Koda M, Okawa A, Aiba A: Transient paraparesis after laminectomy for thoracic ossification of the posterior longitudinal ligament and ossification of the ligamentum flavum. Spinal Cord 44:130-134, 2006

28. Yamazaki M, Mochizuki M, Ikeda Y, Sodeyama T, Okawa A, Koda M, et al: Clinical results of surgery for thoracic myelopathy caused by ossification of the posterior longitudinal ligament: operative indication of posterior decompression with instrumented fusion. Spine (Phila Pa 1976) 31:14521460, 2006

29. Yonenobu K, Ebara S, Fujiwara K, Yamashita K, Ono K, Yamamoto $\mathrm{T}$, et al: Thoracic myelopathy secondary to ossification of the spinal ligament. J Neurosurg 66:511-518, 1987

30. Zhang HQ, Chen LQ, Liu SH, Zhao D, Guo CF: Posterior decompression with kyphosis correction for thoracic myelopathy due to ossification of the ligamentum flavum and ossification of the posterior longitudinal ligament at the same level. J Neurosurg Spine 13:116-122, 2010

\section{Disclosures}

The authors report no conflict of interest concerning the materials or methods used in this study or the findings specified in this paper.

\section{Author Contributions}

Conception and design: Saiwai, Okada, Hayashida, Harimaya, Matsumoto, Kawaguchi, Iwamoto, Nakashima. Acquisition of data: Saiwai, Okada, Hayashida, Harimaya, Matsumoto, Kawaguchi, Kobayakawa, Maeda, Ohta, Shirasawa, Tsuchiya, Terada, Kaji, Arizono, Saito, Fujiwara. Analysis and interpretation of data: Saiwai, Okada, Hayashida, Kobayakawa. Drafting the article: Saiwai, Okada, Hayashida. Approved the final version of the manuscript on behalf of all authors: Saiwai.

\section{Correspondence}

Hirokazu Saiwai: Graduate School of Medical Sciences, Kyushu University, Fukuoka, Japan. hsaiwai@med.kyushu-u.ac.jp. 